\title{
New Answers for Old Questions in the Treatment of Severe Infections from Injection Drug Use
}

\author{
David P Serota, MD, MSc ${ }^{1 *}$, Theresa Vettese, $\mathrm{MD}^{2}$
}

\begin{abstract}
${ }^{1}$ Division of Infectious Diseases, Department of Medicine, University of Miami Miller School of Medicine, Miami, Florida; ${ }^{2}$ Division of General
\end{abstract} Internal Medicine, Department of Medicine, Emory University School of Medicine, Atlanta, Georgia.

Hospitalists are increasingly responsible for the management of infectious consequences of opioid use disorder (OUD), including increasing rates of hospitalization for injection drug use (IDU)-associated infective endocarditis, osteomyelitis, and soft tissue infections. Management of IDU-associated infections poses unique challenges: symptoms of the underlying addiction can interfere with care plans, patients often have difficult psychosocial circumstances in addition to their addiction, and they are often stigmatized by the healthcare system. Although there are few randomized trial data to support one particular approach to management, the literature suggests that successful treatment of IDU-associated infections requires appropriate antimicrobial and surgical interventions in addition to acknowledgment and treatment of the underlying OUD. In this narrative review, the best available evidence is used to answer several of the most commonly encountered questions in the management of IDUassociated infections. These data are used to develop a framework for hospitalists to approach the care of patients with IDU-associated infections. Journal of Hospital Medicine 2020;15:606-612. (C) 2020 Society of Hospital Medicine
A $s$ a result of the epidemic of opioid use disorder (OUD), there has been a secondary surge in hospitalizations for infectious complications of injection drug use (IDU). ${ }^{1,2}$ In the previous 10 years, there have been significant increases in IDU-associated human immunodeficiency virus $(\mathrm{HIV})^{3}$ and hepatitis $\mathrm{C}$ virus $(\mathrm{HCV})^{4}$ infection as well as increased hospitalizations from IDU-associated skin and soft tissue infections, osteomyelitis, septic arthritis, bacteremia, fungemia, and infective endocarditis in the United States. ${ }^{2,5-7}$ Patients admitted with IDU-associated infections have long lengths of stay, high rates of leaving against medical advice (AMA), readmission, and mortality. ${ }^{8-13}$ In a British cohort (median age 36 years), 5 -year mortality after an episode of IDU-associated endocarditis was $42 \% .{ }^{14}$ Admissions for IDU-associated infections can be a troubling experience for both patients and providers alike. ${ }^{15}$ While management decisions of IDU-associated infectious syndromes have sometimes been based on emotion, dogma, and an often-stigmatizing approach toward people suffering from addiction, ${ }^{16}$ with a better understanding of addiction and effective treatments, as well as accumulating data in both addiction and infectious disease fields, it is an appropriate time to reevaluate the approach to treatment.

The goal of this review is to examine recent evidence and attempt to answer questions that frequently arise in the manage-

*Corresponding Author: David P. Serota, MD, MSc; Email: dserota@med. miami.edu; Telephone: 305-905-4214.

Published online first December 18, 2019

Received: June 12, 2019; Revised: October 5, 2019; Accepted: October 5, 2019 ๑ 2020 Society of Hospital Medicine DOI 10.12788/jhm.3342 ment of hospitalized patients with IDU-associated infections. The questions addressed in this review primarily stem from the discussion of Schranz and colleagues in their description of increasing hospitalizations for IDU-associated endocarditis.? Additionally, questions were developed from discussions with practicing academic hospitalists. For each question, a review of the published literature was performed, with a focus on articles published between 2014 and 2019. Finally, a framework for how to approach patients with infectious complications of IDU is presented. As a comprehensive review of infectious complications of OUD would be difficult to cover in one review, this review will focus on eight questions that frequently arise in the care of inpatients.

\section{KEY MANAGEMENT QUESTIONS IN THE INPATIENT MANAGEMENT OF INFECTIOUS COMPLICATIONS OF OUD}

How Should OUD Be Managed in the Hospital?

Management of an IDU-associated infection is incomplete without addressing the underlying addiction in some way. Addiction is highly undertreated among patients with IDU-associated infections, which may contribute to poor infection-related outcomes, ${ }^{8,13,17}$ Opioid agonist therapy (buprenorphine and methadone) to prevent withdrawal should be routinely offered to all patients with OUD, including those with infectious complications of OUD, to facilitate appropriate medical treatment and engage patients in long-term addiction treatment. Referral to addiction treatment has been associated with improved IDU-associated endocarditis mortality, ${ }_{18}^{18}$ and initiation of medications for OUD (MOUD) can be achieved successfully in the emergency department, inpatient wards, and specifically in patients admitted with IDU-associated endocarditis. ${ }^{19-21}$ Protocols and resources for 
inpatient management of withdrawal and initiation of MOUD are available along with telephone support services for providers seeking guidance on specific cases. ${ }^{21,22}$ Inpatient addiction consult services are an important resource for the management of hospitalized patients with addiction and are associated with increased completion of antibiotics, decreased AMA discharge, and increased rates of MOUD provision among patients with IDU-associated infections. ${ }^{12}$ However, when unavailable, initiation of opioid agonist therapy does not require an addiction specialist. Linkage to outpatient addiction care is ideal; however, opioid agonist therapy initiated in the hospital can be tapered prior to discharge if this is unavailable. Figure 1 outlines the initiation of methadone or buprenorphine for the treatment of both withdrawal and OUD in the inpatient setting. ${ }^{20,21}$

\section{Who Can Prescribe Medications for Treatment of OUD in Hospitalized Patients?}

Although buprenorphine prescribing in the outpatient setting requires certification, inpatient physicians are exempt from these requirements and can prescribe buprenorphine or methadone in the hospital setting..$^{20}$ In the outpatient setting, buprenorphine prescription is restricted to providers with a Drug Addiction Treatment Act of 2000 (DATA 2000) waiver, also known as an "X-waiver." $X$-waiver training is 8 hours, and free web-based training is available. ${ }^{23}$ At the time of discharge, non-X-waivered physicians can prescribe up to 72 hours of buprenorphine as a bridge to follow-up with outpatient addiction services. ${ }^{24}$ In the outpatient setting, methadone can only be obtained through approved methadone maintenance programs (MMP); however, many such programs are often willing to do intakes on the same day or next day following hospital discharge. For patients already taking methadone at the time of admission, their MMP should be contacted during business hours to confirm the patient-reported dose. If the MMP cannot be contacted on the day of admission, the starting dose of methadone indicated in Figure 1 is sufficient to prevent precipitation of acute withdrawal. The decision of whether to initiate buprenorphine, methadone, or extended-release naltrexone for the treatment of OUD is nuanced and includes consideration of local resources, patient preference, comorbidities, and hospital policy. Successful initiation of inpatient MOUD requires knowledge of local addiction treatment resources. Social workers and case managers can be used to identify outpatient providers willing to continue MOUD. If no plans or desire for outpatient addiction treatment exist, methadone and buprenorphine can be tapered during the last week of hospitalization.

\section{Is It Safe to Place a Peripherally Inserted Central Catheter in a Patient Who Injects Drugs?}

Many practitioners believe that IDU is an absolute contraindication to the use of peripherally inserted central catheters (PICC) for administration of antimicrobials; however, evidence of harm is lacking. ${ }^{25,26}$ In a review of outpatient parenteral antimicrobial therapy (OPAT) in patients with IDU, there were low overall rates of line-related adverse events and no significant difference in complications between IDU and non-IDU patients receiving OPAT. ${ }^{27}$ As with any medical intervention, risks and benefits must be balanced. Aside from patient comfort, a PICC allows patients to receive intravenous (IV) antimicrobials in a nonhospital setting, which may be more therapeutic for their addiction. Peripheral venous access can be difficult in patients with IDU who often have atrophic superficial veins. While often cited as a reason to avoid PICCs, there is no empirical evidence that PICC placement leads to increased drug use among people with OUD. Similarly, depriving a patient of a PICC does not prevent drug use, but it may prevent patients from completing infection treatment in a more acceptable setting. The most serious concern with a PICC is that if a patient injects drugs, transient bacteremia/fungemia could seed this prosthetic material and lead to worsening infection. Providers should employ a risk-based approach to the use of PICCs considering patient preferences, addiction disease activity, and stability of home environment weighed against the potential risks of prolonged hospitalization, clinic-based antibiotic infusions through a peripheral IV, or possibly suboptimal oral antimicrobial treatment.

\section{What Is the Best Location for Patients to Receive} Antibiotics for Their IDU-Associated Infection?

Antimicrobial treatment for severe IDU-associated infections such as endocarditis and osteomyelitis has traditionally included 4- to 6-week hospital admissions to complete the entirety of IV therapy. This practice has recently been called into question. Extended hospitalization for patients with IDU-associated infections-often not receiving evidence-based treatment for their addiction — can be a harrowing experience and may be antitherapeutic. ${ }^{15,28}$ Disposition decisions for patients with IDU-associated infections should involve risk stratification to assess addiction disease activity and take into account inpatient addiction treatment resources and patient preference, culture/availability of skilled nursing facilities (SNFs), and safety of the home environment. ${ }^{29}$ Some emerging models of care take advantage of long hospitalizations by engaging patients with comprehensive addiction services including substance use group meetings, counseling, and social resources. Another model using OPAT with intensive outpatient follow-up for both addiction and infection treatment showed similar infection outcomes, lower cost, and improved patient satisfaction compared with in-hospital treatment. ${ }^{30}$ When available, medical respite programs and OPAT-friendly residential addiction programs have shown success and financial savings as well. ${ }^{31,32}$ Still, many patients would prefer home OPAT, and there is evidence that home OPAT is no less successful than OPAT provided in an SNF. ${ }^{33}$ Despite this mounting evidence, there remains systemic stigmatization of people with OUD and inequity, as many SNFs and home infusion companies will not provide either MOUD or services to patients with OUD. ${ }^{34}$

\section{Can Oral Antibiotics Be Used to Treat Severe Infections Due to IDU?}

A general principle of infectious diseases is that oral antibiotics should be used whenever possible when presumed to be noninferior to IV alternatives. Accumulating evidence in the infectious disease literature suggests that there is a role for in- 


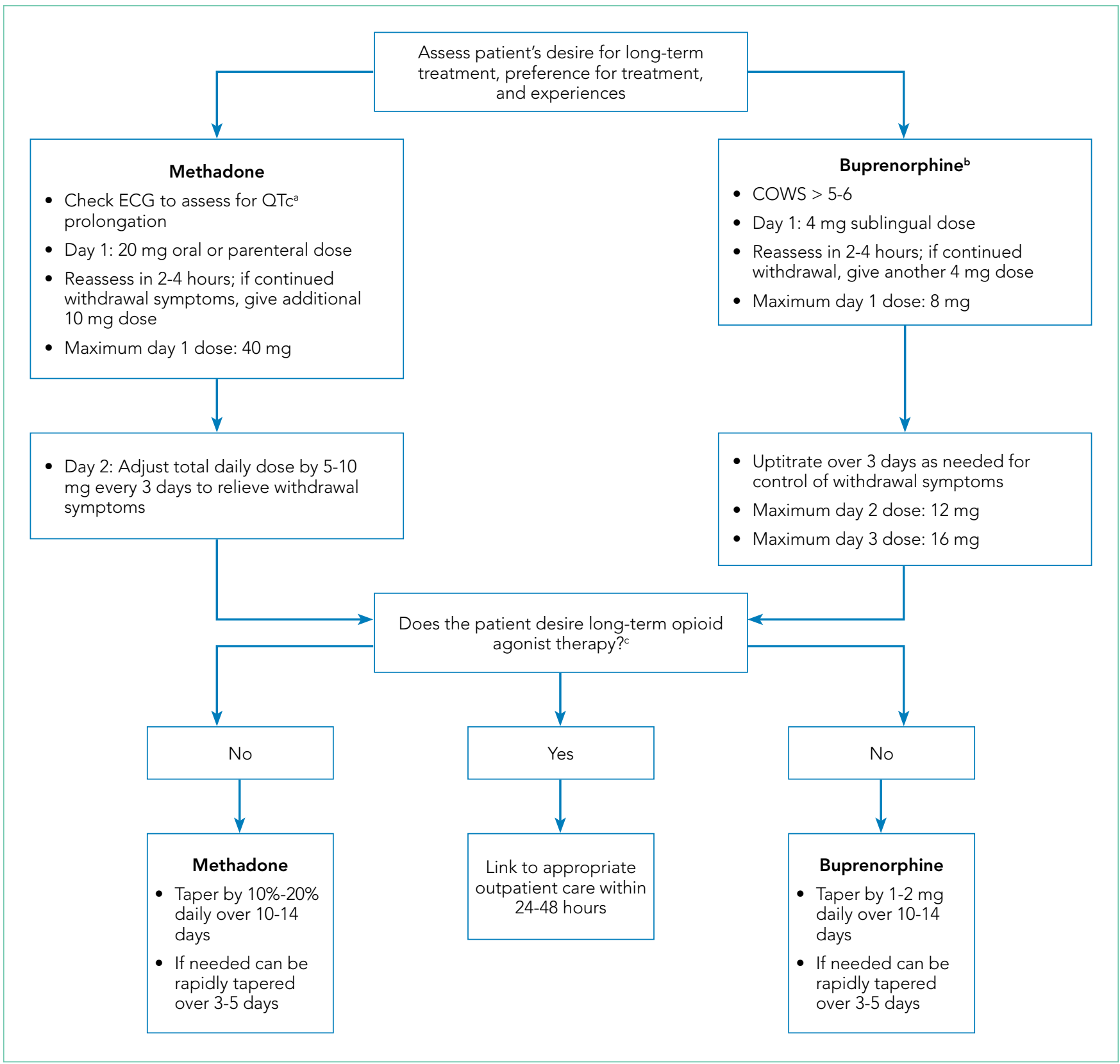

FIG 1. Treatment of Hospitalized Patients with Opioid Use Disorder Undergoing Withdrawal

amethadone should be used with caution in patients with QTc prolongation.

bBuprenorphine can precipitate opioid withdrawal and should only be used in patients already with clear evidence of opioid withdrawal.

cLong-term opioid agonist therapy is preferred and should be strongly encouraged.

Abbreviations: COWS, clinical opioid withdrawal scale; ECG, electrocardiogram; QTc, corrected QT.

creasing the use of oral antibiotics for serious infections. Two recent pivotal randomized trials have questioned the dogma surrounding the use of IV antibiotics for the management of orthopedic infections and endocarditis. However, these studies included few patients with infections due to IDU. 35,36 One study of oral antibiotics specifically in patients with IDU-associated infection showed that an all-oral regimen for the management of IDU-associated right-sided endocarditis was effective and well-tolerated. ${ }^{37}$ While oral antibiotics decrease the need for long-term hospitalization and OPAT, similar or even more intensive follow-up of these patients is required to ensure an appropriate response to treatment. Oral antibiotics should not be used to simply expedite discharge but instead should be done with careful planning and close follow-up.

When using oral antibiotics for severe infections, attempts should be made to use agents with the highest oral bioavailability, tolerability, and affordability. Antimicrobials with near-complete oral bioavailability include fluoroquinolones, triazoles, oxazolidinones (linezolid and tedizolid), clindamycin, trimethoprim-sulfamethoxazole, doxycycline, metronida- 


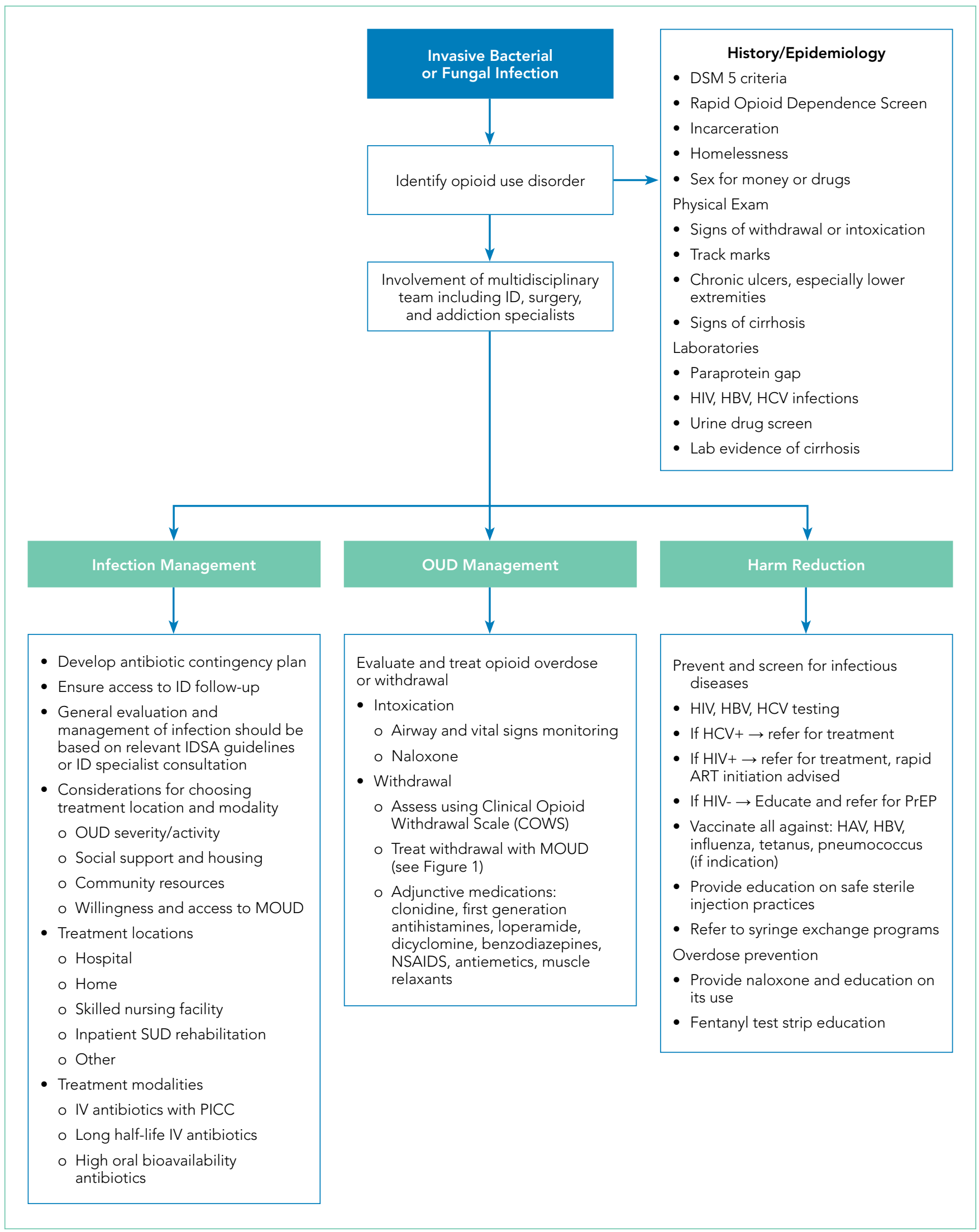

FIG 2. Framework for the Management of Patients with IDU-Associated Infections

Abbreviations: ART, antiretroviral therapy; DSM 5, Diagnostic and Statistical Manual of Mental Disorders 5; HAV, hepatitis A virus; HBV, hepatitis B virus; HCV, hepatitis C virus; HIV, human immunodeficiency virus; ID, infectious diseases; IDSA, Infectious Diseases Society of America; IDU, injection drug use; IE, infective endocarditis; IV, intravenous; MOUD, medications for opioid use disorder; NSAID, nonsteroidal anti-inflammatory drug; OUD, opioid use disorder; PICC, peripherally inserted central catheter; PrEP, HIV preexposure prophylaxis. 
zole, cefadroxil, and other select oral cephalosporins. One approach is to complete a short course of inpatient induction therapy with IV antimicrobials followed by consolidation therapy with oral antibiotics. In a study of uncomplicated Staphylococcus aureus bacteremia, a similar approach with initial IV therapy and oral linezolid follow-up treatment was noninferior to all-IV treatment. ${ }^{38}$ Decisions about the early transition to oral antimicrobials should be made in conjunction with infectious disease specialists where available.

\section{What Is the Role of Long Half-Life IV Antibiotics for Treating IDU-Associated Infections?}

Dalbavancin and oritavancin are extremely long half-life IV glycopeptide antibiotics for gram-positive bacterial infections that require, at most, weekly administration. These agents allow IV-equivalent antibiotics to be delivered without the need for daily infusions or PICCs. Currently, both are approved by the United States Food and Drug Administration only for skin and skin structure infections, but there are increasing reports of successful use in more severe infections including osteomyelitis, bacteremia, and endocarditis. ${ }^{39-42}$ Two studies of dalbavancin in vulnerable populations, including primarily IDU-associated infections, found a somewhat unimpressive $56 \%$ and $71 \%$ clinical response and success rate, respectively. Without comparison groups, one cannot conclude that patients would have done any better with traditional OPAT or long-term hospitalization. ${ }^{24,43}$ Overall, the role of these antimicrobials in IDU-associated infections remains unclear.

\section{Is Surgical Placement of Prosthetic Material Safe in Patients With IDU-Associated Infections?}

When surgery for an IDU-associated infection has the potential to be acutely lifesaving, it should be offered. There is a concern that surgical interventions that require placement of prosthetic material might serve as a nidus of future infection in the setting of ongoing IDU. Although treatments for many substance use disorders are effective-particularly medications to treat OUD_addiction is a relapsing chronic condition, and at least, some future drug use is an expected part of the course. Research comparing outcomes after valve surgery between IDU and non-IDU-associated endocarditis patients shows no difference in short-term outcomes, ${ }^{44}$ but longer-term data show increased mortality between 60 and 180 days postoperatively, higher rates of valve-related complications, and up to $53 \%$ reinfection rates. ${ }^{10,45,46}$ These studies are limited by the lack of a nonsurgically treated control group and little information on the rate of addiction treatment, which may be protective against these negative outcomes. In contrast, another study found that surgery was the strongest predictor of survival among patients with IDU-associated endocarditis after a median of 3.6 years follow-up. ${ }^{18}$ Another consideration is that patients with IDU-associated infection tend to be younger, and despite advancements, many modern prostheses have a finite lifespan. When multiple surgical options exist, a procedure that avoids prosthetic material is preferred. For example, in a meta-analysis of studies of tricuspid valve endocarditis $(41 \%$
IDU-associated), there was no mortality difference between valve repair compared with valve replacement, but there was a significantly lower rate of recurrent endocarditis among those with a repair only. ${ }^{47}$ Decisions about surgery must be individualized and consider a patient's engagement in OUD treatment, social support, prior success with treatment, treatment and relapse prevention resources, and access to harm reduction interventions such as sterile syringes.

What Are Appropriate Harm Reduction Interventions for Patients Hospitalized With Infections Due to IDU? A prolonged admission for IDU-associated infections is an opportunity to provide patients with education, health maintenance services, and secondary prevention interventions for both infection and overdose. Based on epidemiologic risk, patients should be screened for HIV, HCV, hepatitis B, syphilis, gonorrhea, and chlamydia. Patients should be vaccinated against hepatitis $A$, influenza, and tetanus (and pneumococcus if indicated), if unvaccinated or without vaccination records. Patients positive for HIV should be evaluated by an infectious disease specialist with consideration of the rapid initiation of antiretroviral therapy. Patients positive for $\mathrm{HCV}$ or hepatitis $\mathrm{B}$ should be referred for treatment in the outpatient setting. $\mathrm{Pa}$ tients without HIV should be educated about HIV preexposure prophylaxis and referred to outpatient services.

Harm reduction involves meeting patients where they are and providing services they are willing to accept to improve their health or prevent negative outcomes. One important strategy for reducing harm involves maintaining patients in care for their addiction and infection as much as possible, ideally avoiding AMA discharge. In one cohort of patients admitted with IDU-associated infections and OUD, 49\% of those without an addiction medicine consult left AMA. ${ }^{12}$ If patients plan to leave AMA, all efforts should be made to provide them with oral antibiotics that might be effective, even if suboptimal, for their infection. Hospitalists should consider documenting an oral "antibiotic contingency plan" that can be rapidly enacted if a patient is imminently leaving the hospital. The patient should be provided with outpatient follow-up appointments with infectious disease or primary care. All patients with IDU-associated infections should be discharged with naloxone, overdose prevention education, and community resources for addiction treatment and syringe exchange programs.

\section{GENERAL APPROACH TO INPATIENT MANAGEMENT OF INFECTIOUS COMPLICATIONS OF OUD}

Management of IDU-associated infection should be organized around a multidisciplinary framework with careful attention to infection treatment, OUD treatment, and harm reduction interventions (Figure 2). The first step in managing IDU-associated infections is recognizing addiction in the acute care setting. Substance use disorders, including OUD, are often unrecognized in patients presenting with IDU-associated infections. ${ }^{48}$ The Rapid Opioid Dependence Screen, a validated screening tool for OUD, can be quickly administered for all patients who 
present with endocarditis, bacteremia, skin and soft tissue infections, vertebral and epidural infections, and HIV and HCV infections. ${ }^{49}$ In addition to directly questioning patients about substance use, Figure 2 lists epidemiologic, physical exam, and laboratory findings that might suggest to the provider that OUD may be present.

The approach to infection management is similar to non-IDU-associated infections, including identifying a source, evaluating for complications and need for source control procedures, and administering antimicrobials. Management of the substance use disorder includes treatment of acute withdrawal, control of pain, initiation of MOUD when appropriate, and linkage to outpatient addiction treatment services in addition to harm reduction interventions.

\section{CONCLUSION}

Hospital admissions for infectious complications of IDU are increasingly common and are difficult experiences for both patients and providers. However, these hospitalizations serve as a "reachable moment" to engage patients with OUD into medical care and initiate holistic treatment of their infection and underlying substance use disorder. ${ }^{28,50}$ Significant systems-level barriers remain to comprehensive management of the overlapping infectious disease and opioid epidemics. Nevertheless, it is critical to acknowledge that the infections are a symptom of an underlying substance use disorder, a key first step in improving the care of patients hospitalized with infectious complications of OUD. Just like the many acute exacerbations of chronic illness managed by hospitalists, treatment of these episodes of "acute decompensated addiction" require evidence-based management of the underlying disease and its infectious consequences using a harm reduction approach.

Disclosures: The authors both report no conflict of interest.

\section{References}

1. Rudd RA, Seth P, David F, Scholl L. Increases in drug and opioid-involved overdose deaths - United States, 2010-2015. MMWR Morb Mortal Wkly Rep. 2016;65(5051):1445-1452. https://doi.org/10.15585/mmwr.mm655051e1

2. Ronan MV, Herzig SJ. Hospitalizations related to opioid abuse/dependence and associated serious infections increased sharply, 2002-12. Health Aff (Millwood). 2016;35(5):832-837. https://doi.org/10.1377/hlthaff.2015.1424

3. Peters PJ, Pontones P, Hoover KW, et al. HIV infection linked to injection use of oxymorphone in Indiana, 2014-2015. N Engl J Med. 2016;375(3):229-239. https://doi.org/10.1056/NEJMoa1515195

4. Zibbell JE, lqbal $K$, Patel RC, et al. Increases in hepatitis $C$ virus infection related to injection drug use among persons aged $\leq 30$ years - Kentucky, Tennessee, Virginia, and West Virginia, 2006-2012. MMWR Morb Mortal Wkly Rep. 2015;64(17):453-458.

5. Wurcel AG, Anderson JE, Chui KK, et al. Increasing infectious endocarditis admissions among young people who inject drugs. Open Forum Infect Dis. 2016;3(3):ofw157. https://doi.org/10.1093/ofid/ofw157

6. Hartnett $\mathrm{KP}$, Jackson KA, Felsen $\mathrm{C}$, et al. Bacterial and fungal infections in persons who inject drugs - Western New York, 2017. MMWR Morb Mortal Wkly Rep. 2019;68(26):583-586. https://doi.org/10.15585/mmwr.mm6826a2

7. Schranz AJ, Fleischauer A, Chu VH, Wu LT, Rosen DL. Trends in drug use-associated infective endocarditis and heart valve surgery, 2007 to 2017: a study of statewide discharge data. Ann Intern Med. 2019.170(1):31-40. https://doi. org/10.7326/M18-2124

8. Rosenthal ES, Karchmer AW, Theisen-Toupal J, Castillo RA, Rowley CF. Suboptimal addiction interventions for patients hospitalized with injection drug use-associated infective endocarditis. Am J Med. 2016;129(5):481-485. https://doi.org/10.1016/j.amjmed.2015.09.024

9. Rabkin DG, Mokadam NA, Miller DW, Goetz RR, Verrier ED, Aldea GS. Longterm outcome for the surgical treatment of infective endocarditis with a focus on intravenous drug users. Ann Thorac Surg. 2012;93(1):51-57. https:// doi.org/10.1016/j.athoracsur.2011.08.016

10. Kim JB, Ejiofor JI, Yammine M, et al. Surgical outcomes of infective endocarditis among intravenous drug users. J Thorac Cardiovasc Surg. 2016;152(3):832-841.e1. https://doi.org/10.1016/j.jtcvs.2016.02.072

11. Leahey PA, LaSalvia MT, Rosenthal ES, Karchmer AW, Rowley CF. High morbidity and mortality among patients with sentinel admission for injection drug use-related infective endocarditis. Open Forum Infect Dis. 2019;6(4):ofz089. https://doi.org/10.1093/ofid/ofz089

12. Marks LR, Munigala S, Warren DK, Liang SY, Schwarz ES, Durkin MJ. Addiction medicine consultations reduce readmission rates for patients with serious infections from opioid use disorder. Clin Infect Dis. 2019;68(11):19351937. https://doi.org/10.1093/cid/ciy924

13. Serota DP, Niehaus ED, Schechter MC, et al. Disparity in quality of infectious disease vs addiction care among patients with injection drug use-associated Staphylococcus aureus bacteremia. Open Forum Infect Dis. 2019;6(7):ofz289. https://doi.org/10.1093/ofid/ofz289

14. Straw S, Baig MW, Gillott R, et al. Long-term outcomes are poor in intravenous drug users following infective endocarditis, even after surgery. Clin Infect Dis. 2019. https://doi.org/10.1093/cid/ciz869

15. Bearnot BI, Mitton JA, Hayden M, Park ER. Experiences of care among individuals with opioid use disorder-associated endocarditis and their healthcare providers: Results from a qualitative study. J Subst Abuse Treat. 2019;102:1622. https://doi.org/10.1016/j.jsat.2019.04.008

16. Hull SC, Jadbabaie F. When is enough enough? The dilemma of valve replacement in a recidivist intravenous drug user. Ann Thorac Surg. 2014;97(5):1486-1487. https://doi.org/10.1016/j.athoracsur.2014.02.010

17. Jicha C, Saxon D, Lofwall MR, Fanucchi LC. Substance use disorder assessment, diagnosis, and management for patients hospitalized with severe infections due to injection drug use. J Addict Med. 2019;13(1):69-74. https:// doi.org/10.1097/ADM.0000000000000454

18. Rodger L, Glockler-Lauf SD, Shojaei E, et al. Clinical characteristics and factors associated with mortality in first-episode infective endocarditis among persons who inject drugs. JAMA Netw Open. 2018;1(7):e185220. https://doi. org/10.1001/jamanetworkopen.2018.5220

19. Suzuki J. Medication-assisted treatment for hospitalized patients with intravenous-drug-use related infective endocarditis. Am J Addict. 2016;25(3):191194. https://doi.org/10.1111/ajad.12349

20. Theisen-Toupal J, Ronan MV, Moore A, Rosenthal ES. Inpatient management of opioid use disorder: a review for hospitalists. J Hosp Med. 2017;12(5):369374. https://doi.org/10.12788/jhm.2731

21. Donroe JH, Holt SR, Tetrault JM. Caring for patients with opioid use disorder in the hospital. CMAJ. 2016;188(17-18):1232-1239. https://doi.org/10.1503/ cmaj.160290

22. Englander H, Mahoney S, Brandt K, et al. Tools to support hospital-based addiction care: core components, values, and activities of the improving addiction care team. J Addict Med. 2019;13(2):85-89. https://doi.org/10.1097/ ADM.00000000000000487

23. 8 Hour Online MAT Waiver Training. Providers Clinical Support System 2019. Accessed May 22, 2019. https://learning.pcssnow.org/p/onlinematwaiver

24. Bork JT, Heil EL, Berry S, et al. Dalbavancin use in vulnerable patients receiving outpatient parenteral antibiotic therapy for invasive gram-positive infections. Infect Dis Ther. 2019;8(2):171-184. https://doi.org/10.1007/s40121019-0247-0

25. Rapoport AB, Fischer LS, Santibanez S, Beekmann SE, Polgreen PM, Rowley CF. Infectious diseases physicians' perspectives regarding injection drug use and related infections, United States, 2017. Open Forum Infect Dis. 2018;5(7):ofy132. https://doi.org/10.1093/ofid/ofy132

26. Fanucchi L, Leedy N, Li J, Thornton AC. Perceptions and practices of physicians regarding outpatient parenteral antibiotic therapy in persons who inject drugs. J Hosp Med. 2016;11(8):581-582. https://doi.org/10.1002/ jhm.2582

27. Suzuki J, Johnson J, Montgomery M, Hayden M, Price C. Outpatient parenteral antimicrobial therapy among people who inject drugs: a review of the literature. Open Forum Infect Dis. 2018;5(9):ofy194. https://doi.org/10.1093/ ofid/ofy194

28. Velez CM, Nicolaidis C, Korthuis PT, Englander H. "It's been an Experience, a life learning experience": a qualitative study of hospitalized patients with substance use disorders. J Gen Intern Med. 2017;32(3):296-303. https://doi. org/10.1007/s11606-016-3919-4 
29. Eaton EF, Mathews RE, Lane PS, et al. A 9-point risk assessment for patients who inject drugs requiring intravenous antibiotics may allow health systems to focus inpatient resources on those at greatest risk of ongoing drug use. Clin Infect Dis. 2019;68(6):1041-1043. https://doi.org/10.1093/cid/ciy722

30. Fanucchi LC, Walsh SL, Thornton AC, Nuzzo PA, Lofwall MR. Outpatient parenteral antimicrobial therapy plus buprenorphine for opioid use disorder and severe injection-related infections. Clin Infect Dis. 2019.pii:ciz654. https://doi.org/10.1093/cid/ciz654

31. Beieler AM, Dellit TH, Chan JD, et al. Successful implementation of outpatient parenteral antimicrobial therapy at a medical respite facility for homeless patients. J Hosp Med. 2016;11(8):531-535. https://doi.org/10.1002/ jhm.2597

32. Jewell C, Weaver M, Sgroi C, Anderson K, Sayeed Z. Residential addiction treatment for injection drug users requiring intravenous antibiotics: a cost-reduction strategy. J Addict Med. 2013;7(4):271-276. https://doi.org/10.1097/ ADM.0b013e318294b1eb

33. D'Couto HT, Robbins GK, Ard KL, Wakeman SE, Alves J, Nelson SB. Outcomes according to discharge location for persons who inject drugs receiving outpatient parenteral antimicrobial therapy. Open Forum Infect Dis 2018;5(5):ofy056. https://doi.org/10.1093/ofid/ofy056

34. Wakeman SE, Rich JD. Barriers to post-acute care for patients on opioid ag onist therapy; an example of systematic stigmatization of addiction. J Gen Intern Med. 2017;32(1):17-19. https://doi.org/10.1007/s11606-016-3799-7

35. Iversen K, Ihlemann N, Gill SU, et al. Partial oral versus intravenous antibiotic treatment of endocarditis. N Engl J Med. 2019;380(5):415-424. https://doi. org/10.1056/NEJMoa1808312

36. Li HK, Rombach I, Zambellas R, et al. Oral versus intravenous antibiotics for bone and joint infection. N Engl J Med. 2019;380(5):425-436. https://doi. org/10.1056/NEJMoa1710926

37. Dworkin RJ, Lee BL, Sande MA, Chambers HF. Treatment of right-sided Staphylococcus aureus endocarditis in intravenous drug users with ciprofloxacin and rifampicin. Lancet. 1989;2(8671):1071-1073. https://doi.org/10.1016/ s0140-6736(89)91083-0

38. Willekens R, Puig-Asensio M, Ruiz-Camps I, et al. Early oral switch to linezolid for low-risk patients with Staphylococcus aureus bloodstream infections: a propensity-matched cohort study. Clin Infect Dis. 2018. https://doi. org/10.1093/cid/ciy916

39. Rappo U, Puttagunta S, Shevchenko V, et al. Dalbavancin for the treatment of osteomyelitis in adult patients: a randomized clinical trial of efficacy and safety. Open Forum Infect Dis. 2018;6(1):ofy331. https://doi.org/10.1093/ ofid/ofy331
40. Morata L, Cobo J, Fernandez-Sampedro M, et al. Safety and efficacy of prolonged use of dalbavancin in bone and joint infections. Antimicrob Agents Chemother. 2019;63(5).pii. e02280-18. https://doi.org/10.1128/AAC.02280-18

41. Tobudic S, Forstner C, Burgmann H, et al. Dalbavancin as primary and sequential treatment for gram-positive infective endocarditis: 2-year experience at the General Hospital of Vienna. Clin Infect Dis. 2018;67(5):795-798. https://doi.org/10.1093/cid/ciy279

42. Wunsch $S$, Krause R, Valentin $T$, et al. Multicenter clinical experience of real life dalbavancin use in gram-positive infections. Int J Infect Dis. 2019;81:210214. https://doi.org/10.1016/j.ijid.2019.02.013

43. Bryson-Cahn C, Beieler AM, Chan JD, Harrington RD, Dhanireddy S. Dalbavancin as secondary therapy for serious Staphylococcus aureus infections in a vulnerable patient population. Open Forum Infect Dis. 2019;6(2):ofz028. https://doi.org/10.1093/ofid/ofz028

44. Hall R, Shaughnessy M, Boll G, et al. Drug-use and post-operative mortality following valve surgery for infective endocarditis: a systematic review and meta-analysis. Clin Infect Dis. 2019;69(7):1120-1129. https://doi.org/10.1093/ cid/ciy 1064

45. Shrestha NK, Jue J, Hussain ST, et al. Injection drug use and outcomes after surgical intervention for infective endocarditis. Ann Thorac Surg. 2015;100(3):875-882. https://doi.org/10.1016/j.athoracsur.2015.03.019

46. Osterdal OB, Salminen PR, Jordal S, Sjursen H, Wendelbo O, Haaverstad R. Cardiac surgery for infective endocarditis in patients with intravenous drug use. Interact Cardiovasc Thorac Surg. 2016;22(5):633-640. https://doi. org/10.1093/icvts/ivv397

47. Yanagawa B, Elbatarny M, Verma S, et al. Surgical management of tricuspid valve infective endocarditis: a systematic review and meta-analysis. Ann Thorac Surg. 2018;106(3):708-714. https://doi.org/10.1016/j.athoracsur.2018.04.

48. Miller AC, Polgreen PM. Many opportunities to record, diagnose, or treat injection drug-related infections are missed: a population-based cohort study of inpatient and emergency department settings. Clin Infect Dis. 2019;68(7):116-1175. https://doi.org/10.1093/cid/ciy632

49. Wickersham JA, Azar MM, Cannon CM, Altice FL, Springer SA. Validation of a brief measure of opioid dependence: the rapid opioid dependence screen (RODS). J Correct Health Care. 2015;21(1):12-26. https://doi. org/10.1177/1078345814557513

50. Englander H, Collins D, Perry SP, Rabinowitz M, Phoutrides E, Nicolaidis C. "We've learned it's a medical illness, not a moral choice": qualitative study of the effects of a multicomponent addiction intervention on hospital providers' attitudes and experiences. J Hosp Med. 2018;13(11):752-758. https://doi. org/10.12788/jhm.2993 